CTP TAMU-5/95

Imperial/TP/94-95/21

hep-th/9502108

\title{
Embedding of the Bosonic String into the $W_{3}$ String
}

\author{
H. Lü̈円, C.N. Pope*1, K.S. Stelle ${ }^{\$}$ and K.W. Xu*1 \\ * Center for Theoretical Physics, Texas A\&M University, College Station, TX 77843-4242 \\ \$ The Blackett Laboratory, Imperial College, Prince Consort Road, London SW7 2BZ
}

\begin{abstract}
We investigate new realisations of the $W_{3}$ algebra with arbitrary central charge, making use of the fact that this algebra can be linearised by the inclusion of a spin- 1 current. We use the new realisations with $c=102$ and $c=100$ to build non-critical and critical $W_{3}$ BRST operators. Both of these can be converted by local canonical transformations into a BRST operator for the Virasoro string with $c=28-2$, together with a Kugo-Ojima topological term. Consequently, these realisations provide embeddings of the Virasoro string into non-critical and critical $W_{3}$ strings.
\end{abstract}

\footnotetext{
${ }^{1}$ Supported in part by the U.S. Department of Energy, under grant DE-FG05-91-ER40633

${ }^{2}$ Supported in part by the Commission of the European Communities under contracts SC1*-CT91-0674 and SC1*-CT92-0789.
} 


\section{Introduction}

The properties of a string theory are determined by the realisation of its underlying world-sheet symmetry algebra. Owing to the non-linearity of the $W_{3}$ algebra, which makes realisations hard to come by, the corresponding $W_{3}$ strings have only been extensively studied in the freescalar realisations found by Romans [1]. In this type of realisation, one of the scalar fields plays a distinguished rôle, and the remainder occur only in the form of their energy-momentum tensor. It is therefore of interest to look for new realisations that could be used to build other $W_{3}$ strings. Some progress has recently been made in understanding the structure of the $W_{3}$ algebra. It has been shown that if the $W_{3}$ algebra is extended to $W_{1+3}$ by including a spin-1 current, then after performing a non-linear basis change this can be recast into the form of a linear algebra [2]. After this basis change, the spin-3 current becomes a null current, and the linear $W_{1+3}$ algebra is generated by this null current, an energy-momentum tensor, and the spin-1 current. The procedure can be reversed. If the null current is set to zero, one obtains precisely a Romans-type realisation of the $W_{3}$ algebra with the derivative of the distinguished scalar field replaced by the abstract spin-1 current. However, it was shown in Ref. [2] that the null current of the $W_{1+3}$ algebra can be also be realised by a non-vanishing parafermionic vertex operator. In section 2 , we obtain new realisations in which the null current is realised with ghost-like fields. The central charge of the resulting $W_{3}$ realisations can be arbitrary. An unusual feature of these realisations is that the spin-3 current commutes with itself at the classical level. The $W_{3}$ symmetry is effectively absent at the classical level (i.e. at the level of single contractions in OPEs); it arises properly as a consequence of quantisation.

The new realisation takes its simplest form when $c=102$. In section 3 , we shall use this realisation to build a $W_{3}$ string. Since the critical central charge for the $W_{3}$ algebra is $c=100$, we shall need to make use of the non-critical BRST operator for $W_{3}$ that was constructed in [3]. In this construction, a nilpotent BRST operator is obtained by taking two abstract realisations of the $W_{3}$ algebra, with the requirement that their total central charge adds up to $c=100$. One of the two realisations can be viewed as the "Liouville" sector and the other as the "matter" sector. We shall use the new realisation of the $W_{3}$ algebra for the matter sector. Then we need a $c=-2$ realisation for the Liouville sector. Interestingly enough, this is the unique value of the central charge for which the $W_{3}$ algebra can be realised by a single scalar field. Alternatively, one can fermionise this scalar and realise the algebra using a pair of fermions $\left(b_{1}, c_{1}\right)$, of spins 1 and 0 respectively. We shall show that the resulting BRST operator can be re-expressed, by means of a similarity transformation, as a BRST operator for a Virasoro string with central charge $c=28-2$, together with a purely topological Kugo-Ojima term whose only non-trivial operator is the identity. Thus, the cohomology of this $W_{3}$ string is entirely equivalent to that of the usual Virasoro string. This provides an embedding of the Virasoro string into a non-critical $W_{3}$ string.

Another way to use the $c=102$ realisation to build a nilpotent BRST operator is to gauge also the spin-1 current, since the ghosts for the additional current make a $c=-2$ contribution to the central charge. Once the spin-1 current is included, the constraints become reducible. The resulting nilpotent BRST operator for $W_{1+3}$ is equivalent to that for a Virasoro string with central charge $c=28-2$, as can be seen again by performing a similarity transformation. This implies that the non-critical $W_{3}$ BRST operator for the new $c=102$ realisation can be viewed as equivalent to a critical $W_{1+3}$ BRST operator, with the fields $\left(b_{1}, c_{1}\right)$ of the Liouville sector of the non-critical BRST operator being reinterpreted as the ghosts for the spin-1 current of the 
critical BRST operator.

In section 4 , we shall give the details of how to construct a critical $W_{3}$ BRST operator, using the new $W_{3}$ realisation at $c=100$. After making a local similarity transformation, we shall show that the BRST operator is equivalent to that for the $c=28-2$ Virasoro string. This gives the embedding of the Virasoro string into the critical $W_{3}$ string mentioned above. This also proves that, in the new realisation, the $c=102-2$ non-critical $W_{3}$ string is equivalent to a $c=100$ critical $W_{3}$ string.

It is also of interest to examine the problem of embedding the Virasoro string into extended string theories in a more general context. In addition to the embedding into the non-critical $W_{3}$ string mentioned above, in section 2 and section 3 we shall also examine the embedding into a $W_{2, s}$ string, whose world-sheet symmetry algebra is generated by a spin- $s$ current in addition to the energy-momentum tensor. In particular, we shall construct explicit embeddings of the Virasoro string for the cases $s=1,2,3$.

\section{The linear $W_{1+3}$ algebra and new realisations for $W_{3}$}

We begin by reviewing the linearisation of the $W_{3}$ algebra by the inclusion of a spin-1 current [2]. The operator-product expansion of the spin- 1 current with the spin- 2 current $T$ and spin-3 current $W$ of the $W_{3}$ algebra is uniquely determined by requiring that the Jacobi identities be satisfied. The resulting $W_{1+3}$ algebra can then be turned into a linear algebra by a non-linear invertible basis change:

$$
\begin{aligned}
T_{0} & =T, \\
W_{0} & =W+\frac{1}{3}(J J J)+\frac{1}{2}(J T)+\frac{3+2 t^{2}}{2 t}\left(J \partial J+\frac{1}{4} \partial T+\frac{\left(3+2 t^{2}\right)}{6 t} \partial^{2} J\right), \\
J_{0} & =J,
\end{aligned}
$$

where the $T, W$ currents generate the $W_{3}$ algebra with central charge

$$
c=50+16 t^{2}+\frac{36}{t^{2}},
$$

and the OPEs $J(z) W(w)$ and $J(z) T(w)$ are uniquely determined by the Jacobi identities [2]. (For simplicity, we have rescaled the $W$ current relative to the usual normalisation.) In this new basis, the OPEs of the $W_{1+3}$ algebra become linear:

$$
\begin{aligned}
T_{0}(z) T_{0}(0) & \sim \frac{c}{2 z^{4}}+\frac{2 T_{0}}{z^{2}}+\frac{\partial T_{0}}{z}, \quad T_{0}(z) W_{0}(0) \sim \frac{3 W_{0}}{z^{2}}+\frac{\partial W_{0}}{z} \\
T_{0}(z) J_{0}(0) & \sim-\frac{3 / t+2 t}{z^{3}}+\frac{J_{0}}{z^{2}}+\frac{\partial J_{0}}{z}, \quad J_{0}(z) J_{0}(0) \sim-\frac{1}{z^{2}} \\
J_{0}(z) W_{0}(0) & \sim \frac{t W_{0}}{z}, \quad W_{0}(z) W_{0}(0) \sim 0 .
\end{aligned}
$$

Note that in the linear basis, the spin-3 current has become the null current $W_{0}$. The invertibility of the non-linear basis change (1) implies that we can reverse the procedure, and obtain a realisation of the $W_{3}$ algebra with central charge (2) in terms of $W_{0}, T_{0}$ and $J_{0}$. If the null current $W_{0}$ is set to zero, one obtains a Romans-type realisation of the $W_{3}$ algebra, with the derivative of the distinguished scalar replaced by the abstract spin-1 current $J_{0}$. If the null 
current $W_{0}$ is non-zero, however, one obtains a new realisation. The non-vanishing current $W_{0}$ was first realised in Ref. [2] using parafermionic vertex operators. In this paper, we shall instead realise the $W_{0}$ current with ghost-like fields. We first consider such a realisation of the $W_{1+3}$ algebra (3), given by

$$
T_{0}=T_{X}+2 \partial r s+3 r \partial s, \quad W_{0}=r, \quad J_{0}=r s,
$$

where $(r, s)$ are a pair of bosonic ghost-like fields with the OPE $r(z) s(w) \sim 1 /(z-w)$. It is easy to see from the $J_{0}(z) W_{0}(w)$ OPE that $t=-1$. It follows from Eqn. (2) that $T_{0}$ must have central charge $c=102$, and hence from Eqn. (团) that the central charge for the energymomentum tensor $T_{X}$ is $c_{X}=28$.

Having found a realisation of the linear $W_{1+3}$ algebra, we may now invert the basis change (11) to obtain a new realisation of the $W_{3}$ algebra with central charge $c=102$ :

$$
\begin{aligned}
T & =T_{X}+2 \partial r s+3 r \partial s \\
W & =r-\frac{1}{3} r^{3} s^{3}-\frac{1}{2} r s T_{X}+\frac{5}{2} \partial r r s^{2}+\frac{1}{8} r \partial^{2} s+\frac{1}{8} \partial r \partial s-\frac{7}{4} \partial^{2} r s+\frac{5}{8} \partial T_{X} .
\end{aligned}
$$

An unusual feature of this realisation, which contrasts with the situation in the usual freescalar realisations, is that the classical terms in the $W$ current, i.e. $r-\frac{1}{3} r^{3} s^{3}$, commute with themselves at the classical level. [3 $^{3}$ Thus, at the classical level, the realisation (5) generates just a Virasoro algebra together with a null spin-3 current. It is only at the quantum level that the $W_{3}$ symmetry properly manifests itself. Note that, although the realisation (4) for $W_{1+3}$ is reducible, since $J_{0}=s W_{0}$, the corresponding realisation (5) of $W_{3}$ is not reducible. It is worth remarking that $T$ and $W$ still generate the $W_{3}$ algebra with $c=102$ even when the first term, $r$, is absent from $W$. In that case, one has a Romans-type realisation, with the derivative of the distinguished scalar replaced by $J_{0}=r s$. If we bosonise the $(r, s)$ fields, $r=\partial \xi e^{-\phi}$ and $s=\eta e^{\phi}$, then the $(\xi, \eta)$ fields and $T_{X}$ appear in the $W$ current in (5) only via the energy-momentum tensor $T$ in Eqn. (5). Then one has precisely a Romans realisation, with $\phi$ as the distinguished scalar together with an effective energy-momentum tensor $T_{X}+T_{\xi \eta}=T_{X}-\eta \partial \xi$. The field $r$ can have arbitrary spin in this case with the $r$ term absent from $W$, i.e. when $W_{0}=0$. The inversion of the basis change (1) then gives rise to a realisation of the $W_{3}$ algebra at arbitrary central charge.

The new formulation of the $W_{3}$ string that we present in this paper is based on a realisation including the $r$ term in the $W$ current. The fact that the realisation (5) has central charge $c=102$ is a consequence of our choice of the realisation (团) for $W_{1+3}$. We can construct new realisations, which generalise the one given in Eqn. (4), to obtain new realisations of $W_{3}$ with arbitrary central charge. To do this, we introduce a pair of ghost-like anti-commuting fields $\left(b_{1}, c_{1}\right)$ with spin $(k, 1-k)$. The realisations of $W_{1+3}$ with arbitrary central charge take the form

$$
\begin{aligned}
T_{0} & =T_{X}+2 \partial r s+3 r \partial s-k b_{1} \partial c_{1}-(k-1) \partial b_{1} c_{1}, \\
W_{0} & =r \\
J_{0} & =-t r s+\sqrt{t^{2}-1} b_{1} c_{1}
\end{aligned}
$$

\footnotetext{
${ }^{3}$ In this paper, we assign the dimension of the fields $\left(b, c, b_{1}, c_{1}, \phi, X^{\mu}\right)$ to be $\left(\hbar, \hbar^{0}, \hbar, \hbar^{0}, \hbar^{\frac{1}{2}}, \hbar^{\frac{1}{2}}\right)$ respectively. For the $(r, s)$ and $(\beta, \gamma)$ fields, whose spins $(j, 1-j)$ change with context, we assign the dimensions to be $\left(\hbar^{j}, \hbar^{1-j}\right)$.

${ }^{4}$ One can also bosonise the $(r, s)$ fields differently, $r=e^{i \rho+\phi}$ and $s=-i \partial \rho e^{-i \rho-\phi}$. In that case, the $W_{0}$ current is a pure exponential, which is equivalent to a special case of the parafermionic realisations discussed in Ref. [2].
} 
where $k=\frac{1}{2}+\frac{3}{2} t^{-1} \sqrt{t^{2}-1}$. Note that the $\left(b_{1}, c_{1}\right)$ fields here can be bosonised and hence replaced by a free scalar. The central charge of the realisation is given by (2). When $t^{2}=1$, the $b_{1}$ and $c_{1}$ fields do not appear in $J_{0}$, the central charge is $c=102$, and $k=\frac{1}{2}$. This gives rise to the $W_{1+3}$ realisation (四), with $T_{X}$ replaced by $T_{X}+T_{b_{1} c_{1}}$. If one chooses $t^{2}=\frac{9}{8}$ instead, the central charge is $c=100$ and $k=1$.

Having obtained the realisations (6) of the $W_{1+3}$ algebra, it is straightforward to obtain realisations of $W_{3}$ with arbitrary central charge simply by inverting the basis change (11). In particular, when one chooses the central charge parameter $t^{2}=\frac{9}{8}$, one obtains a realisation of the $W_{3}$ algebra at $c=100$. In section 4 , we shall construct the critical $W_{3}$ BRST operator for this realisation and study its cohomology. Before doing so, we shall construct in section 3 the non-critical BRST operator for the realisation (5) with $c=102$.

\section{The non-critical $W_{3}$ BRST operator and its cohomology}

In this section, we use the $c=102$ realisation (5) to construct a $W_{3}$ string. To do this, we must combine it with a $c=-2$ realisation to form a non-critical BRST operator as constructed in [3]. We shall use a pair of fermions $\left(b_{1}, c_{1}\right)$ with spins $(1,0)$ to form the $c=-2$ realisation of $W_{3}$ 叫:

$$
T_{\mathrm{L}}=-b_{1} \partial c_{1}, \quad W_{\mathrm{L}}=\partial b_{1} \partial c_{1}-b_{1} \partial^{2} c_{1} .
$$

Note that this realisation is reducible, and that it does not close classically but it does close at the quantum level. This reducibility is harmless, however, since this realisation enters only in the Liouville sector of the theory. By a local canonical field redefinition, the BRST operator can be transformed into a graded form [5]

$$
\begin{aligned}
Q= & Q_{0}+Q_{1} \\
Q_{0}= & \oint c\left(T-b_{1} \partial c_{1}-2 \partial \beta \gamma-3 \beta \partial \gamma-b \partial c\right) \\
Q_{1}= & \oint \gamma\left(W+\frac{3}{2} b_{1} c_{1} T-\frac{9}{2} b_{1} c_{1} \beta \partial \gamma-38 \partial T\right. \\
& \left.+\frac{21}{4} b_{1} \partial^{2} c_{1}+6 \partial b_{1} \partial c_{1}+\frac{15}{4} \partial^{2} b_{1} c_{1}+\frac{3}{4} \partial \beta \partial \gamma\right),
\end{aligned}
$$

where $(c, b)$ and $(\gamma, \beta)$ are ghosts and anti-ghosts for the spin-2 and spin-3 currents respectively, and $T$ and $W$ are given in Eqn. (5). The effective energy-momentum tensor $T_{X}$ that appears in the operator $Q_{1}$ can be removed by a similarity transformation $Q \longrightarrow e^{R} Q e^{-R}$, with $R$ given by

$$
R=\oint \frac{3}{2} b\left(\gamma b_{1} c_{1}+\gamma r s+\frac{3}{2} \partial \gamma\right) .
$$

The BRST operator $Q$ in (8) can then be re-expressed as

$$
\begin{aligned}
Q= & Q_{0}+Q_{1} \\
Q_{0}= & \oint c\left(T_{X}+2 \partial r s+3 r \partial s-b_{1} \partial c_{1}-2 \partial \beta \gamma-3 \beta \partial \gamma-b \partial c\right), \\
Q_{1}= & \oint \gamma\left(r-\frac{1}{3} r^{3} s^{3}+\frac{7}{2} \partial r r s^{2}+\frac{3}{2} r^{2} \partial s s-\frac{1}{2} r s b_{1} \partial c_{1}-\frac{3}{2} r s \beta \partial \gamma\right. \\
& \left.-\frac{9}{4} \partial^{2} r s-\partial r \partial s+\frac{1}{4} r \partial^{2} s+\frac{1}{2} b_{1} \partial^{2} c_{1}+\frac{3}{4} \partial b_{1} \partial c_{1}+\frac{5}{4} \partial \beta \partial \gamma\right) .
\end{aligned}
$$


It is interesting to note that the Liouville sector $\left(b_{1}, c_{1}\right)$ enters the operator $Q_{1}$ only as a quantum correction to the classical terms $\gamma\left(r-\frac{1}{3} r^{3} s^{3}\right)$. The BRST operator $Q$ remains nilpotent even if the term $\gamma r$ is absent. If one were to bosonise the $(r, s)$ fields in that case, the BRST operator would become the same as the one given in Ref. [6], except that in this paper we use Eqn. (7) rather than a two-scalar realisation for the $c=-2$ Liouville sector, and use $T_{X}+T_{\xi \eta}$ for the $c=26$ effective energy-momentum tensor. However, as we shall see later, the inclusion of the $\gamma r$ term is crucial for obtaining a genuine embedding of the Virasoro string into the non-critical $W_{3}$ string.

The BRST operator (10) for the $W_{3} \equiv W_{2,3}$ case can easily be generalised to the cases of $W_{2,1}$ and $W_{2,2}$ strings. For the $W_{2,1}$ string, the BRST operator is given by

$$
\begin{aligned}
Q_{0} & =\oint c\left(T_{X}+r \partial s-b_{1} \partial c_{1}-\beta \partial \gamma-b \partial c\right), \\
Q_{1} & =\oint \gamma\left(r-r s-b_{1} c_{1}\right) .
\end{aligned}
$$

For the $W_{2,2}$ case, it is given by

$$
\begin{aligned}
Q_{0} & =\oint c\left(T_{X}+\partial r s+2 r \partial s-b_{1} \partial c_{1}-\partial \beta \gamma-2 \beta \partial \gamma-b \partial c\right) \\
Q_{1} & =\oint \gamma\left(r-\frac{1}{2} r^{2} s^{2}+r \partial s+2 \partial r s-b_{1} \partial c_{1}-\beta \partial \gamma\right) .
\end{aligned}
$$

There should be no confusion from the fact that the $(r, s)$ and $(\beta, \gamma)$ fields have different conformal spins in different BRST operators. It is easy to verify that the $(r, s)$ terms in $\left\{Q_{1}, \beta\right\}$, for $Q_{1}$ given in (12), realise the Virasoro algebra with central charge $c=28$.

Having constructed the non-critical BRST operator for the $c=102$ realisation (5) of $W_{3}$, we now would like to study its cohomology. In fact, we shall show that the BRST operator given in (10) provides an embedding of the Virasoro string into the non-critical $W_{3}$ string. As we shall see later, the BRST operators given in (11), (12) and (10) can all be re-expressed, by local similarity transformations, in the form

$$
\begin{aligned}
Q_{0} & =\oint c\left(T_{X}+(j-1) \partial r s+j r \partial s-b_{1} \partial c_{1}-(j-1) \partial \beta \gamma-j \beta \partial \gamma-b \partial c\right), \\
Q_{1} & =\oint \gamma r
\end{aligned}
$$

for $j=1,2,3$ respectively. This BRST operator can be simplified further by the similarity transformation $Q \longrightarrow e^{R} Q e^{-R}$, with $R$ given by

$$
R=\oint s(c \partial \beta+j \partial c \beta),
$$

leading to

$$
Q_{0}=\oint c\left(T_{X}-b_{1} \partial c_{1}-b \partial c\right), \quad Q_{1}=\oint \gamma r .
$$

Since the $(\beta, \gamma)$ and $(r, s)$ fields form a Kugo-Ojima quartet [8], the BRST operator (15) simply describes a $c=26$ dimensional Virasoro string with an energy-momentum tensor given by $T_{X}-b_{1} \partial c_{1}$. 
In the above similarity transformations, the most difficult part is the transformation of the BRST operators (11), (12) and (10) into the form given in (13). We shall discuss this procedure case by case. First, let us consider the $W_{2,1}$ BRST operator given in (11). The similarity transformation involves an infinite number of terms, given by

$$
R=\sum_{n \geq 1} R_{n}=-\oint \sum_{n \geq 1} \frac{2^{1-n}}{n(n+1)}\left(r s^{n+1}+(n+1) b_{1} c_{1} s^{n}\right) .
$$

It is easy to see that this similarity transformation converts the BRST operator (11) into the form (13) with $j=1$. Since $R$ is primary under the total energy-momentum tensor, and since it does not contain the $(b, c)$ ghosts, this transformation leaves the operator $Q_{0}$ invariant. That $Q_{1}$ is converted into the form $\gamma r$ is a consequence of the fact that $e^{-R} r e^{R}=r-r s-b_{1} c_{1}$. Note that all the terms in Eqn. (16) are classical. This is a special feature for the $W_{2,1}$ case, which does not occur for higher-spin cases. The absence of quantum corrections here occurs simply because they are all total derivatives when $j=1$.

Next we consider the $W_{2,2}$ case, whose BRST operator is given in (12). In this case, the full similarity transformation is very complicated. It is instructive to study the transformation first at the classical level. The classical terms of the $Q_{1}$ operator are $\gamma\left(r-\frac{1}{2} r^{2} s^{2}\right)$. At the classical level, there is an invertible local canonical transformation of the $(r, s)$ fields that converts the classical terms into $\gamma r$, namely

$$
\begin{aligned}
& r \longleftarrow r-\frac{1}{2} r^{2} s^{2}, \\
& s \longleftarrow \sum_{n \geq 0} g_{n} r^{n} s^{2 n+1},
\end{aligned}
$$

where $g_{n}=n(2 n+1)^{-1} g_{n-1}$ with $g_{0}=1$. It is easy to verify that this canonical transformation leaves the associated energy-momentum tensor appearing in the $Q_{0}$ operator (12) invariant at the classical level. The classical terms of the generator $R$ associated with the transformation (17) are more complicated. They take the form

$$
R_{\mathrm{cl}}=\sum_{n \geq 1} R_{n}=\oint \sum_{n \geq 1} h_{n} r^{n+1} s^{2 n+1} .
$$

The first few coefficients are given by $h_{1}=\frac{1}{6}, h_{2}=\frac{1}{60}, h_{3}=\frac{41}{15120}, h_{4}=\frac{1}{1890}, h_{5}=\frac{3337}{29937600}$, etc. In order to obtain the generator $R$ at the full quantum level, it is also necessary to add quantum corrections to the classical terms (18). Although the $R$ operator involves infinitely many terms, it is possible to construct it in a systematic way since the terms can be organised according to their grading degree under the $(r, s)$ number operator $J=r s$. In fact, the classical terms of $R_{n}$ in Eqn. (18) have degree $n$. For each $R_{n}$, there is only a finite number of candidates for possible quantum corrections. Since the similarity transformation leaves $Q_{0}$ invariant, $R_{n}$ has to satisfy $\left[R_{n}, T^{\text {tot }}\right]=0$, with $T^{\text {tot }}=\left\{Q_{0}, b\right\}$. Furthermore, it has to satisfy the following recursive conditions organised by the $(r, s)$ grading:

$$
\begin{aligned}
{\left[R_{1}, \gamma r\right] } & =Q_{1}^{\prime}, \\
{\left[R_{2}, \gamma r\right]-\frac{1}{2}\left[R_{1},\left[R_{1}, \gamma r\right]\right] } & =0 \\
{\left[R_{3}, \gamma r\right]-\frac{1}{2}\left[R_{1},\left[R_{2}, \gamma r\right]\right]-\frac{1}{2}\left[R_{2},\left[R_{1}, \gamma r\right]\right]+\frac{1}{6}\left[R_{1},\left[R_{1},\left[R_{1}, \gamma r\right]\right]\right] } & =0
\end{aligned}
$$


where $Q_{1}^{\prime}$ is $Q_{1}$ in Eqn. (12) with the $\gamma r$ term removed. The computation can be simplified by noting that the classical terms in $R_{n}$ also have degree zero under the $\left(b_{1}, c_{1}\right)$ and $(\beta, \gamma)$ number operators. By making the ansatz that this property persists at the quantum level, the number of candidate terms for each $n$ is greatly reduced. $R_{1}$ can easily be solved, and is given by

$$
R_{1}=\oint s\left(\frac{1}{6} r^{2} s^{2}+\frac{3}{2} r \partial s+\beta \partial \gamma+\frac{1}{2} \partial \beta \gamma+b_{1} \partial c_{1}\right) .
$$

Note that the general forms of the quantum corrections can be obtained by making the replacements $r s \rightarrow \partial$, or $r^{2} s^{2} \rightarrow b_{1} c_{1} \partial$, etc. The numbers of possible quantum corrections in $R_{n}$ and the complexity of the computations increase rapidly with $n$. We have solved the recursive conditions (19) explicitly for $R_{1}, R_{2}$ and $R_{3}$. It is worth remarking that all the coefficients in $R_{1}$ are fixed by the recursive conditions (19). There are two free parameters in $R_{2}$ and 10 free parameters in $R_{3}$. Although we have not completely analysed the recursive conditions (19) for higher values of $n$, we expect that the solutions exist and that the number of free parameters increases with $n$. The fact that solutions of this form can be found justifies the simplifying ansatz made above.

Having obtained the similarity transformations for the $W_{2,1}$ and $W_{2,2}$ cases, it is straightforward to discuss the $W_{2,3}$ case. The similarity transformation generator here is even more complicated again. As in the cases discussed above, it is instructive first to study the transformation at the classical level. The classical terms of the operator $Q_{1}$ given in (10) are $\gamma\left(r-\frac{1}{3} r^{3} s^{3}\right)$. They can be transformed classically into $\gamma r$ by the invertible local field redefinition

$$
\begin{aligned}
& r \longleftarrow r-\frac{1}{3} r^{3} s^{3}, \\
& s \longleftarrow \sum_{n \geq 0} g_{n} r^{2 n} s^{3 n+1},
\end{aligned}
$$

where $g_{n}=n(3 n+1)^{-1} g_{n-1}$ and $g_{0}=1$. This field redefinition leaves the operator $Q_{0}$ in (10) invariant. The terms of the similarity transformation generator $R$ for the classical transformation (21) take the form

$$
R_{\mathrm{cl}}=\sum_{n \geq 1} R_{n}=\oint \sum_{n \geq 1} h_{n} r^{2 n+1} s^{3 n+1},
$$

where $h_{1}=\frac{1}{12}, h_{2}=\frac{1}{168}, h_{3}=\frac{43}{60480}, h_{4}=\frac{41}{393120}, h_{5}=\frac{26917}{1585059840}$, etc. Analogously to the $W_{2,2}$ case, the $R_{n}$ term in Eqn. (22) has a grading degree $n$ under the $(r, s)$ number operator $J=r s$, and has degree zero under the $\left(b_{1}, c_{1}\right)$ and $(\beta, \gamma)$ number operators. Assuming this grading structure persists at the quantum level, we can consider the possible quantum corrections to $R_{n}$. In addition to the requirement that $\left[R_{n}, T^{\text {tot }}\right]=0$, with $T^{\text {tot }}=\left\{Q_{0}, b\right\}, R_{n}$ has to satisfy the recursive conditions organised by the $(r, s)$ grading, as given in (19), where $Q_{1}^{\prime}$ is now the operator $Q_{1}$ given in (10) with the $\gamma r$ term absent. The complexity of solving these recursive conditions increases dramatically with $n$. We have solved explicitly for $R_{1}$ and $R_{2}$. As in the $W_{2,2}$ case, the number of free parameters in $R_{n}$ increases with $n$.

While we have not given in closed form the full quantum-level canonical transformations needed to cast the BRST operator into the form of Eqn. (13), some aspects of the closed-form expression can nevertheless be outlined. At the classical level, the transformation (21) maps $(r, s)$ to $(\tilde{r}, \tilde{s})$, where

$$
\begin{aligned}
& \tilde{r}=r\left(1-\frac{1}{3} x\right), \\
& \tilde{s}=s g(x),
\end{aligned}
$$


where $x=r^{2} s^{3}$. The transformation (23) is canonical provided the Poisson brackets for $r$ and $s$ are preserved, which requires that the function $g(x)$ satisfy the differential equation

$$
x(3-x) \frac{d}{d x} g(x)+(1-x) g(x)=1 .
$$

This differential equation can be solved:

$$
g(x)=\frac{k+\int^{x} d y y^{-2 / 3}\left(1-\frac{1}{3} y\right)^{-1 / 3}}{3 x^{1 / 3}\left(1-\frac{1}{3} x\right)^{2 / 3}},
$$

where $k$ is an integration constant. The integral in (25) yields a hypergeometric function. Methods for promoting such classical canonical transformations to the quantum level in $d=1$ quantum mechanical systems are now well developed [9], and one may anticipate the applicability of such methods to the quantum forms of transformations like Eqns. (17) and (21) in $d=1+1$ conformal field theory as well.

To end this section, we recall that Eqn. (4) gives a critical realisation of the $W_{1+3}$ algebra (3). Thus, one can construct a nilpotent BRST operator for the system. The constraints are then reducible, however, since $J_{0}=s W_{0}$, and therefore the spin-1 gauge symmetry in this system is effectively fictitious. This can also be seen from the BRST operator:

$$
Q=\oint\left(c\left(T_{0}-3 \beta \partial \gamma-2 \partial \beta \gamma-b_{1} \partial c_{1}-b \partial c\right)+\gamma W_{0}+c_{1} J_{0}-c_{1} \beta \gamma\right) .
$$

After inserting the realisation (4), one can use a similarity transformation with $R=\oint s(c \partial \beta+$ $3 \partial c \beta-\beta c_{1}$ ) to convert the BRST operator (26) into the form of Eqn. (15), where the ghost fields $\left(b_{1}, c_{1}\right)$ originally corresponding to the spin-1 current can now be reinterpreted as a Liouville sector. Thus, in this realisation the non-critical $W_{3}$ string is equivalent to a critical $W_{1+3}$ string.

\section{The critical $W_{3}$ BRST operator and its cohomology}

Now we construct a critical BRST operator using the new realisation of the $W_{3}$ algebra at $c=100$. As discussed in section 2 , the new realisation can be obtained from the $W_{1+3}$ realisation (6) by inverting the basis change (1). By making a canonical field redefinition [7], we find that the corresponding critical BRST operator can be written in a graded form, given by

$$
\begin{aligned}
Q_{0}= & \oint c\left(T_{X}+2 \partial r s+3 r \partial s-b_{1} \partial c_{1}-2 \partial \beta \gamma-3 \beta \partial \gamma-b \partial c\right) \\
Q_{1}= & \oint \gamma\left(r-\frac{1}{3} r^{3} s^{3}+\frac{1}{3} r^{2} s^{2} b_{1} c_{1}+\frac{10}{3} \partial r r s^{2}+\frac{4}{3} r^{2} \partial s s-\frac{2}{3} r s b_{1} \partial c_{1}\right. \\
& -\frac{8}{9} r s \partial b_{1} c_{1}-\frac{4}{3} r s \beta \partial \gamma-\frac{55}{27} \partial^{2} r s-\frac{20}{27} \partial r \partial s+\frac{8}{27} r \partial^{2} s-\frac{10}{9} \partial r s b_{1} c_{1} \\
& \left.-\frac{4}{9} r \partial s b_{1} c_{1}+\frac{1}{9} b_{1} \partial^{2} c_{1}+\frac{4}{9} \partial b_{1} \partial c_{1}+\frac{10}{27} \partial^{2} b_{1} c_{1}+\frac{4}{9} \beta \partial \gamma b_{1} c_{1}+\frac{28}{27} \partial \beta \partial \gamma\right),
\end{aligned}
$$

where $T_{X}$ is an energy-momentum tensor with central charge $c=28$.

In fact, this BRST operator can also be converted into the form of Eqn. (13) by a local similarity transformation. It then follows that its cohomology is equivalent to that of the Virasoro string, with an energy-momentum tensor $T_{X}-b_{1} \partial c_{1}$. Thus, this BRST operator provides an embedding of the $c=28-2$ Virasoro string into the critical $W_{3}$ string. To see 
this, as in the case of the non-critical $W_{3}$ BRST operator discussed in the previous section, it is instructive to study first the similarity transformation at the classical level. The classical terms of the $Q_{1}$ operator (27) are $\gamma\left(r-\frac{1}{3} r^{3} s^{3}+\frac{1}{3} r^{2} s^{2} b_{1} c_{1}\right)$. They can be transformed classically into a single term $\gamma r$ by the invertible local transformation

$$
\begin{aligned}
r & \longleftarrow \\
s & \longleftarrow \sum_{n \geq 0} g_{n} r^{2 n} s^{3 n+1}, \\
b_{1} & \longleftarrow \sum_{n \geq 0} f_{n} r^{2 n} s^{3 n} b_{1}, \\
c_{1} & \longleftarrow \sum_{n \geq 0} e_{n} r^{2 n} s^{3 n} c_{1},
\end{aligned}
$$

where $g_{n}=n(3 n+1)^{-1} g_{n-1}, f_{n}=\frac{1}{9}(3 n-4) n^{-1} f_{n-1}, e_{n}=\frac{1}{9}(3 n-2) n^{-1} e_{n-1}$ and $g_{0}=f_{0}=$ $e_{0}=1$. This transformation leaves the operator $Q_{0}$ in Eqn. (27) invariant. The terms of the operator $R$ that generate this classical-level transformation are given by

$$
R_{\mathrm{cl}}=\sum_{n \geq 1} R_{n}=\oint \sum_{n \geq 1} h_{n}\left(r^{2 n+1} s^{3 n+1}-\frac{1}{3}(3 n+1) r^{2 n} s^{3 n} b_{1} c_{1}\right),
$$

where the $h_{n}$ have precisely the same values as those given below Eqn. (22). Analogously to the case of the non-critical $W_{3}$ BRST operator, one can then obtain the similarity transformation at the quantum level, again organised by the $(r, s)$ grading. Owing to the complexity of the computation, we have solved explicitly only for $R_{1}$ and $R_{2}$.

In this and the previous sections, we have used the new realisations to construct critical and non-critical $W_{3}$ BRST operators. Both of these provide embeddings of the $c=28-2$ Virasoro string. It is also of interest to compare the properties of the two BRST operators. First, we note that $Q_{0}$ is the same for each case. Also, both BRST operators are nilpotent even in the absence of the $\gamma r$ term in $Q_{1}$. When the $\gamma r$ term is included they are equivalent, since both of them can be converted into (15) by local canonical transformations. When the $\gamma r$ term is absent, the two BRST operators become inequivalent. Neither of them is then related to (15) by a local canonical transformation. When the $\gamma r$ term is omitted, it is convenient to consider the BRST operators with $(r, s)$ and $\left(b_{1}, c_{1}\right)$ expressed in bosonised form

$$
\begin{aligned}
r & =\partial \xi e^{-\phi}, \quad s=\eta e^{\phi}, \\
b_{1} & =e^{-i \rho}, \quad c_{1}=e^{i \rho} .
\end{aligned}
$$

In terms of the scalar fields $\phi$ and $\rho$, the non-critical BRST operator becomes the same as the one constructed in [6], except that in this paper we have realised the $c=-2$ Liouville sector by the single scalar $\rho$ rather than by a two-scalar system. The critical BRST operator, on the other hand, is identical to that of a $c=100$ Romans realisation, with the distinguished scalar $\phi_{1}$ given by $(3 \phi+i \rho) / \sqrt{8}$, and the effective energy-momentum tensor $T^{\text {eff }}$ given by $T_{X}-\frac{1}{2}\left(\partial \phi_{2}\right)^{2}-\sqrt{7 / 24} \partial^{2} \phi_{2}$, where $\phi_{2}=(i \phi-3 \rho) / \sqrt{8}$. It is easy to see that $T^{\mathrm{eff}}$ then generates the Virasoro algebra with $c=25 \frac{1}{2}$.

The inequivalence of the two BRST operators with the $\gamma r$ term omitted can be seen in a more general context by studying the most general possible forms of the $Q_{1}$ operator that can be constructed with two scalar fields. We have found that, even at the classical level, there are 
precisely two inequivalent solutions satisfying the nilpotency conditions. Both of these can be extended to the full quantum level. One corresponds to the non-critical $W_{3}$ BRST operator; the other corresponds to the critical $W_{3}$ BRST operator. We have showed above that for both of these BRST operators one can add a term $\gamma r$ after replacing one of the scalars by an $(r, s)$ system, and with this inclusion the critical and non-critical constructions become equivalent, and are also equivalent to an embedding of the Virasoro string.

\section{Conclusions and discussion}

In this paper, we have constructed a new realisation of the $W_{3}$ algebra, making use of the fact that the $W_{3}$ algebra can be linearised through the addition of a spin-1 current. The spin-3 current $W_{0}$ in the linearised $W_{1+3}$ algebra is null. The previously known free-scalar realisation of $W_{3}$ corresponds to the case where this null current is identically zero. The null current, however, can be non-zero and was first realised using parafermionic vertex operators in Ref. [2]. In this paper the null current is realised by ghost-like fields. The central charge of the resulting $W_{3}$ realisation can be arbitrary. In particular, we constructed the cases with central charge $c=102$ and $c=100$, and we built the corresponding non-critical and critical $W_{3}$ string BRST operators. We showed that both BRST operators can be converted into the form of a BRST operator for the Virasoro string with $c=28-2$, together with a Kugo-Ojima term. Thus, the $c=28-2$ Virasoro string can be embedded into both the critical and the non-critical $W_{3}$ strings using the new realisation. Since the Kugo-Ojima system has the identity as its only non-trivial operator, one may consider these to be genuine embeddings, not requiring any further truncations of states. The embedding of the $c=26$ Virasoro string into strings with enlarged worldsheet symmetries was first discussed in Ref. [10], where its embedding into the $N=1$ and $N=2$ superstrings was given.

The embedding of the Virasoro string into the critical $W_{3}$ string has also been previously discussed from a different point of view. The standard Romans realisation of $W_{3}$ at $c=100$ can be viewed in a loose sense as an embedding of the $c=25 \frac{1}{2}$ Virasoro string coupled to the Ising model, since physical states of the $W_{3}$ string are the tensor product of $c=25 \frac{1}{2}$ string states with primary operators of the Ising model. This description of the embedding is not totally satisfactory, however, since the $Q_{1}$ operator, which in this case describes the Ising model, is not purely topological. Later, Ref. [11] proposed an embedding of the $d=26$ Virasoro string into the critical $W_{3}$ string with the usual Romans multi-scalar realisation. However, it had already been established that the cohomology of the multi-scalar $W_{3}$ string corresponds to a $c=25 \frac{1}{2}$ bosonic string coupled to the Ising model, and this is inequivalent to the cohomology of the $d=26$ bosonic string. No local transformation of the field variables can alter this result. Indeed, the similarity transformation used in Ref. [11] to relate the $W_{3}$ string to the $d=26$ Virasoro string involved non-local terms. Such a transformation does not establish an equivalence of the cohomologies of two BRST operators, since the notion of BRST non-triviality must be defined with respect to a given set of local operators. The embeddings described in this paper are genuine, in the sense that they make use only of local canonical transformations in order to establish the equivalence of the BRST cohomologies.

In closing, it is worth remarking that the operator $Q_{1}$ for the $W_{2,2}$ string given in Eqn. (12) is itself a BRST operator for the Virasoro algebra, with $(\beta, \gamma)$ viewed as the ghosts for the energy-momentum tensor. We showed in section 3 that this BRST operator can be converted 
into the single term $\gamma r$, for which the only non-trivial cohomology is the identity. Thus, this can be viewed as the BRST operator for an embedding of an "empty" string into the bosonic string.

\section{Acknowledgements}

H.L., C.N.P. and K.S.S. would like to thank SISSA for hospitality at the beginning of this work. K.S.S. would also like to thank Texas A\&M University for hospitality at its conclusion.

\section{Note Added}

After this paper was completed, we learned of a paper by F. Bastianelli and N. Ohta [12], which has some overlap with our work. They introduced an additional pair of ghost-like bosonic fields $(\tilde{r}, \tilde{s})$ which have spins $(1,0)$, and constructed a $c=102$ realisation of the $W_{1+3}$ algebra with $W_{0}=r$ and $J=r s+\tilde{r}$. They then obtained a $c=102$ realisation of $W_{3}$ and used it to construct a non-critical $W_{3}$ BRST operator. However, they did not obtain the cohomology of the BRST operator.

\section{References}

[1] L.J. Romans, Nucl. Phys. B352 (1991) 829.

[2] S.O. Krivonos and A.S. Sorin, Phys. Lett. B335 (1994) 45.

[3] M. Berschadsky, W. Lerche, D. Nemeschansky and N.P. Warner, Phys. Lett. B292 (1992) 35; Nucl. Phys. B401 (1993) 304.

[4] H. Lü, C.N. Pope, X. Shen and X.J. Wang, Phys. Lett. B267 (1991) 356.

[5] E. Bergshoeff, H.J. Boonstra, S. Panda, M. de Roo and A. Sevrin, Phys. Lett. B308 (1993) 34.

[6] E. Bergshoeff, H.J. Boonstra and M. de Roo, On realising the bosonic string as a noncritical $W_{3}$-string, UG-7/94, hep-th/9409186.

[7] H. Lü, C.N. Pope, S. Schrans and X.J. Wang, Nucl. Phys. B408 (1993) 3.

[8] T. Kugo and I. Ojima, Phys. Lett. B73 (1978) 459.

[9] A. Anderson, Phys. Lett. B319 (1993) 157; Phys. Rev. D47 (1993) 4458; Ann. Phys. 232 (1994) 292; A. Anderson, B.E.W. Nilsson, C.N. Pope and K.S. Stelle, Nucl. Phys. B430 (1994) 107.

[10] N. Berkovits and C. Vafa, Mod. Phys. Lett. A9 (1994) 653.

[11] N. Berkovits, M.D. Freeman and P.C. West, Phys. Lett. B325 (1994) 63.

[12] F. Bastianelli and N. Ohta, Note on $W_{3}$ realisation of the bosonic strings hep-th/9411156, NBI-HE-94-51, OU-HET 203. 